A collaboration between the JAOA and the American Association of Colleges of Osteopathic Medicine (AACOM) to recruit, peer review, publish, and distribute research and other scholarly articles related to osteopathic medical education.

\title{
JAOA/AACOM
}

\section{Correlation of Personal Experience and Acquired Knowledge With Intent to Recommend Adjunctive Osteopathic Manipulative Treatment or Yoga for Patients With Chronic Low Back Pain}

Michael A. Seffinger, DO; Eric Hurwitz, DC, PhD; John Quiamas, DO, MSc; Antoinette Kitch, OMS IV; Vanessa Mervyn-Cohen, DO; Edward Lin, DO

From the Western University of Health Sciences College of Osteopathic Medicine of the Pacific in Pomona, California (Dr Seffinger and Student Doctor Kitch); the University of Hawaii in Honolulu (Dr Hurwitz); Kent Hospital in Warwick, Rhode Island (Dr Quiamas); the Institute for Family Health in New York, New York (Dr Mervyn-Cohen); and Riverside University Health System in Palm Springs, California (Dr Lin). Drs Quiamas and Lin are second-year residents.

Disclaimer: Dr Seffinger, an associate editor of The Journal of the American Osteopathic Association, was not involved in the editorial review or decision to publish this article.

Financial Disclosures: None reported. Support: The study was funded by an educational research grant by the American Association of Colleges of Osteopathic Medicine.

Address correspondence to Michael A. Seffinger, DO, Western University

of Health Sciences College of

Osteopathic Medicine of the Pacific, Department of NMM/OMM, $309 \mathrm{E}$ 2nd St at College Plaza, Pomona, CA 91766-1854. February 16, 2018;

accepted April 3, 2018.
Context: Osteopathic manipulative treatment (OMT) and yoga are both recommended by systematic reviews in the evidence-based research literature for low back pain management. It is unknown, to the authors' knowledge, what the effect of personal experience with OMT or yoga, reading research articles on OMT or yoga, or both will have on medical students' recommendations for these treatment options to future patients with chronic low back pain.

Objective: To evaluate the likelihood of osteopathic medical students recommending OMT or yoga to treat patients with chronic low back pain based on their personal experience or reading research articles that recommend OMT or yoga for patients with chronic low back pain.

Methods: In this prospective cohort study, researchers administered an anonymous 18-question online survey for osteopathic medical students. The survey included a patient vignette, 2 evidence-based articles, and multiple choice, yes/no, and Likerttype questions. Participants were recruited via email from all 4 years of medical school. Between-group differences in proportions were assessed with descriptive statistics and $\chi^{2}$ tests; differences within groups were assessed with the McNemar test; and Fischer exact tests were used when expected cell counts were less than 5.

Results: A total of 180 participants (100 male, 80 female) completed the study. Personal experience increased the likelihood of osteopathic medical students recommending OMT $(P<.018)$ or yoga $(P<.001)$ to a future patient or to a patient in a case vignette $(P<.05)$ with chronic low back pain. Students who read research articles were more likely to recommend OMT to the case patient and future patients before and after reading the intervention article regardless of their experience $(P<.001)$.

Conclusion: Personal experience and reading evidence-based research may increase the likelihood that osteopathic medical students will recommend OMT to future patients with chronic low back pain.

J Am Osteopath Assoc. 2018;118(11):738-745

doi:10.7556/jaoa.2018.159

Keywords: chronic pain, low back pain, OMT, yoga 
$\mathrm{F}$ or the past 2 decades, there has been an increase in the use of adjunctive therapy by patients with low back pain (LBP) and other chronic pain syndromes. ${ }^{1,2}$ This shift in the use of health-related dollars by consumers in part prompted the development of what eventually became the National Center for Complementary and Integrated Health (NCCIH) to provide scientific context to healing practices outside of conventional medicine., By 2015, half of the US medical schools incorporated a Complementary and Alternative Medicine (CAM) course into their curriculum, echoing the population's expansion of traditional medical care. ${ }^{5}$ Medical students agree that exposure to different therapeutic modalities in medical school is important. ${ }^{6}$

What leads a physician to incorporate integrative medicine into his or her practice? The surveys Integrative Medicine Attitude Questionnaire (IMAQ) and CAM Health Belief Questionnaire (CHBQ) were created in an attempt to detect and synthesize health professionals' points of view regarding integrative medicine. ${ }^{7,8}$ The CHBQ includes questions about yoga and osteopathic manipulative treatment (OMT), but IMAQ does not include questions about yoga. ${ }^{9,10}$ Both the CHBQ and the IMAQ assess health care professionals' attitudes toward the principles of integrative medicine and some of its therapies, but they fail to directly assess possible factors that affect the recommendation of integrative therapies to patients.

Both OMT and yoga are interventions that may help treat patients with chronic LBP. In population surveys administered by the NCCIH, OMT and yoga are defined as "complementary and alternative" modalities and are included as forms of integrative therapy. ${ }^{2}$ Additionally, both have been recommended by the $\mathrm{NCCIH}$ as components of a treatment plan for patients with LBP. Basic and clinical science research articles and clinical practice guidelines support the use of both of these modalities for patients with LBP. ${ }^{11-22}$

No studies have assessed what influences medical students' intention to recommend adjunctive therapeutic modalities once they become physicians, to our knowledge. Are personal experience and reading the literature about a modality influential? Since osteopathic medical students already experience and read about OMT in osteopathic medical school, would they embrace another modality, such as yoga, if they experienced it or read about its benefits?

The 4 null hypotheses of this study are as follows:

1. The experience of OMT will have no effect on the likelihood that osteopathic medical students will recommend OMT to future patients.

2. Reading evidence-based research articles on the use of OMT for LBP will have no effect on the likelihood that osteopathic medical students will recommend OMT to future patients.

3. The experience and practice of yoga will have no effect on the likelihood that osteopathic medical students will recommend yoga to future patients.

4. Reading evidence-based research articles on the use of yoga for LBP will have no effect on the likelihood that osteopathic medical students will recommend yoga to future patients.

\section{Methods}

This prospective cohort study was conducted in 2013 and was considered exempt from institutional review board review at Western University of Health Sciences College of Osteopathic Medicine of the Pacific.

\section{Participants}

Osteopathic medical students of all 4 years who were currently enrolled in courses at the Pomona or Lebanon campus were eligible to participate in the study. Participants were recruited via email invitation, with a total of 3 emails sent to the entire student body (1 per week). The link to the computer-generated survey was distributed via email to students who agreed to participate. No financial compensation was provided for participation in the study. 


\section{Design}

The survey used a nonexperimental design of 18 questions created for osteopathic medical students. There were 3 types of answer options: multiple choice, yes/ no, and Likert-type, in which 1 indicated very unlikely and 5 , very likely. This survey attempted to analyze how experience affects the likelihood of osteopathic medical students to recommend OMT or yoga to future patients based on students' experience with these modalities, as well as their knowledge after reading peer-reviewed research articles that recommend the use of these approaches. Participants were asked to read 2 evidence-based articles on the use of $\mathrm{OMT}^{20,23}$ and yoga ${ }^{11,14}$ to improve chronic LBP. The same investigative question was asked before and after the evidencebased articles were read. Additionally, participants were requested to read a clinical vignette of a patient with chronic LBP to further assess student opinion regarding the likelihood of offering a recommendation of OMT or yoga for the sample patient.

\section{Statistical Analysis}

Statistical analysis was performed using Excel 2010 (Microsoft Corporation) and SAS 9.3 (SAS Institute). Descriptive statistics (frequencies and percentages) and $\chi^{2}$ tests were used to analyze the data and test for between-group differences in proportions; McNemar tests were used to test for differences within groups. Fischer exact tests were used when expected cell counts were less than 5. A significance value of $\alpha=.05$ was adopted.

\section{Results}

A total of 180 students of the invited 1320 completed the survey for a response rate of $13.6 \%$ (100 male, 80 female). Osteopathic medical students in all 4 years participated in the study (first-year, 48 [26.7\%]; second-year, 50 [27.8\%]; third-year, 38 [21.1\%]; and fourth-year, 44 [24.4]). No further demographic data were collected. Of the participants, 165 (92\%) had used OMT on patients, $144(80 \%)$ had previous yoga experience, $94(52 \%)$ reported that they currently read research articles about OMT, and $14(8 \%)$ reported that they currently read research articles about yoga. Participants who reported that they currently read research on either modality were termed "readers." "Likely" and "very likely" responses were combined when analyzing overall likelihood to recommend a modality. Analysis showed that experience with the respective modality was more powerful than reading research articles on each respective modality regarding likelihood to recommend them to a patient with chronic LBP (Table 1).

While controlling for experience, reading research articles on OMT increased the proportion of participants who were "very likely" to recommend OMT to the patient in the vignette ( $81 \%$ after reading vs $52 \%$ before; $P<.001)$. Further comparisons of "likeliness" responses showed that participants who had experience using OMT were more likely to recommend OMT to future patients with chronic LBP (93\% with experience vs $73 \%$ without; $P=.018$ ). Participants without experience who read the research articles on OMT were more likely to recommend OMT ( $73 \%$ before reading to $80 \%$ after reading); however, this increase was not statistically significant $(P=.206)$. Readers remained significantly more likely to recommend OMT to patients with LBP before and after reading the 2 articles compared with nonreaders $(82 \%$ reader vs $50 \%$ nonreader before reading articles $[P<.001]$; $81 \%$ reader vs $48 \%$ nonreader after reading articles $[P<.001]$, respectively).

Regardless of experience with yoga, reading research articles about yoga increased the proportion of participants who were likely to recommend yoga $(50 \%$ before reading vs $67 \%$ after among those without experience $[P=.070] ; 78 \%$ before reading vs $87 \%$ after among those with experience $[P=.007])$. Reading research articles increased the proportion of participants who were very likely to recommend yoga to the patient in the vignette, but this increase was not statistically significant ( $43 \%$ after reading vs $28 \%$ before; $P=.06$ ). Respondents who had experience with yoga were more likely to recommend yoga to patients with chronic LBP ("likeliness": $78 \%$ with experience vs $50 \%$ without; $P=.001$ ). 
Table 1.

Likelihood of Participants Recommending OMT and Yoga to Patients With Chronic LBP Before and After Reading Evidence-Based Articles ${ }^{\mathbf{a}}(\mathbf{N}=180)$

\begin{tabular}{|c|c|c|c|c|c|c|}
\hline \multirow[b]{2}{*}{ Participant Characteristic } & \multicolumn{3}{|c|}{$\begin{array}{c}\text { Before Reading } \\
\text { Evidence-Based Articles }\end{array}$} & \multicolumn{3}{|c|}{$\begin{array}{c}\text { After Reading } \\
\text { Evidence-Based Articles }\end{array}$} \\
\hline & Very Likely & Likely & Likeliness & Very Likely & Likely & Likeliness \\
\hline Recommend OMT & $120(67)$ & $44(24)$ & $164(91)$ & $117(65)$ & $46(26)$ & $163(91)$ \\
\hline With experience ${ }^{b}$ & $114(69)$ & $39(24)$ & $153(93)$ & $110(67)$ & $41(25)$ & $151(92)$ \\
\hline Without experience & $6(40)$ & $5(33)$ & $11(73)$ & $7(47)$ & $5(33)$ & $12(80)$ \\
\hline Reader $^{\mathrm{b}}$ & $77(82)$ & $12(13)$ & $89(95)$ & $76(81)$ & $12(13)$ & $88(94)$ \\
\hline Nonreader $^{\mathrm{b}}$ & $43(50)$ & $32(37)$ & $75(87)$ & $41(48)$ & $34(39)$ & $75(87)$ \\
\hline Recommend Yoga & $50(28)$ & $80(44)$ & $130(72)$ & $61(34)$ & $86(48)$ & $147(82)$ \\
\hline With experience ${ }^{b}$ & $47(33)$ & $65(45)$ & $112(78)$ & $56(39)$ & $67(46)$ & $123(85)$ \\
\hline Without experience ${ }^{\mathrm{b}}$ & $3(8)$ & $15(42)$ & $18(50)$ & $5(14)$ & $19(53)$ & $24(67)$ \\
\hline Reader & $7(50)$ & $5(36)$ & $12(86)$ & $6(43)$ & $7(50)$ & $13(93)$ \\
\hline Nonreader & $43(26)$ & $75(45)$ & $118(71)$ & $55(33)$ & $79(48)$ & $134(81)$ \\
\hline $\begin{array}{l}\text { Patient Vignette } \\
\text { Recommendation of OMT }\end{array}$ & $94(52)$ & $58(32)$ & $152(84)$ & $146(81)$ & $22(12)$ & $168(93)$ \\
\hline $\begin{array}{l}\text { Patient Vignette } \\
\text { Recommendation of Yoga }\end{array}$ & 64 (35) & $75(42)$ & $139(77)$ & $77(43)$ & $65(36)$ & $142(79)$ \\
\hline
\end{tabular}

a Data are given as No. (\%).

b Statistically significant $(P<.05)$.

Abbreviation: LBP, low back pain; OMT, osteopathic manipulative treatment.

Participants with experience remained more likely to recommend yoga after reading research articles, but both groups increased in likeliness $(87 \%$ with experience after reading vs $67 \%$ without experience after reading; $P=.004)$. Although not statistically significant, readers of current yoga research were more likely to recommend yoga than were nonreaders $(86 \%$ readers vs $71 \%$ nonreaders; $P=.139)$. Although not statistically significant, reading yoga research articles increased the likelihood that both reader and nonreader groups would recommend yoga and attenuated the difference between the 2 groups ( $93 \%$ readers after reading vs $81 \%$ nonreaders after reading; $P=.493$ ).

The effect on recommendation of a positive or negative experience with OMT or yoga was assessed. There were correlations between having negative experiences with either modality and intent to recommend: With OMT, participants with negative experiences were $54 \%$ less likely to recommend OMT as participants with positive experiences $(P<.000)$; with yoga, participants with negative experiences were half as likely to recommend yoga as participants with positive experiences $(P=.010)$. There were no significant associations between current practice with either OMT or yoga and likelihood of prescribing them.

Overall, participants with experience with OMT or yoga were more likely to recommend its use to the patient in the vignette $(P<.05$; Table 2$)$. Of the 2 modalities, OMT was twice as likely as yoga to be recommended regardless of experience $(80 \%$ OMT vs $40 \%$ yoga by those with experience and $40 \%$ OMT vs $20 \%$ yoga by those without experience; $P<.05$ ). 
Table 2.

Likelihood of Participants Recommending OMT or Yoga in a Patient Vignette With Chronic LBP by Experience With OMT or Yoga and Readership Status of OMT or Yoga Articles $(N=180)^{a}$

\begin{tabular}{lrrr} 
Participant Characteristic by Recommendation & Very Likely & Likely & Likeliness \\
\hline Recommendation of OMT & & & \\
\hline Total sample & $121(67)$ & $39(22)$ & $160(89)$ \\
\hline With experience $(\mathrm{n}=165)$ & $115(70)$ & $32(19)$ & $147(89)$ \\
\hline Negative experience $(\mathrm{n}=9)$ & $1(11)$ & $1(11)$ & $2(22)$ \\
\hline Without experience $(\mathrm{n}=15)$ & $6(40)$ & $7(47)$ & $13(87)$ \\
\hline Reader $(\mathrm{n}=94)$ & $76(81)$ & $11(12)$ & $87(93)$ \\
\hline Nonreader $(\mathrm{n}=86)$ & $45(52)$ & $28(33)$ & $73(85)$ \\
\hline Recommendation of Yoga & & & $139(77)$ \\
\hline Total sample & $64(36)$ & $75(42)$ & $119(83)$ \\
\hline With experience $(\mathrm{n}=144)$ & $57(40)$ & $62(43)$ & $4(40)$ \\
\hline Negative experience $(\mathrm{n}=10)$ & $1(10)$ & $3(30)$ & $20(56)$ \\
\hline Without experience $(\mathrm{n}=36)$ & $7(19)$ & $13(36)$ & $11(79)$ \\
\hline Reader $(\mathrm{n}=14)$ & $6(43)$ & $5(36)$ & $128(77)$ \\
\hline Nonreader $(\mathrm{n}=166)$ & $58(35)$ & $70(42)$ &
\end{tabular}

a Data are given as No. (\%).

Abbreviations: LBP, low back pain; OMT, osteopathic manipulative treatment.

Additionally, reading research articles on OMT increased the amount of participants who were "very likely" to recommend yoga to the patient in the vignette, though this increase was not statistically significant ( $43 \%$ after reading vs $28 \%$ before; $P=.06$ ). Reading research articles about yoga did not have the same effect $(P=.39$ for OMT; $P=.832$ for yoga).

\section{Discussion}

To the authors' knowledge, this is the first study that has assessed whether personal experience with OMT or yoga and/or reading of research articles on OMT or yoga influences osteopathic medical students' likelihood to recommend OMT or yoga for their future patients with chronic LBP. These findings support personal experience as a powerful correlative to the likelihood of recommendation to use OMT or yoga as a component of the treatment plan for patients with chronic LBP. Furthermore, the likelihood of recommendation to use OMT or yoga increased when the participants read research articles.

Osteopathic medical students were more likely to recommend OMT than yoga to a patient with chronic LBP regardless of experience and regardless of whether both are supported by evidence-based articles to help relieve chronic LBP; therefore, other factors may affect the decision to recommend modalities, such as the requirement of learning OMT as part of their medical education curriculum, as well as a requirement to demonstrate proficiency in OMT in order to graduate, receive the DO degree, and obtain a license to practice as an osteopathic physician or surgeon. This finding supplements the findings of a 2011 study $^{24}$ that investigated the relationship between previous experience with OMT and OMT recommendation; 
other factors were found to influence this decision in addition to experience.

In the current study, experience with OMT influenced participants' likelihood of recommending it to the patient in the vignette or future patients with chronic LBP. In the field, however, OMT is not prescribed by osteopathic physicians nearly as much as medication is, even though it is common practice for physicians to prescribe drugs that they have not personally taken. There is clearly more involved in what influences an osteopathic medical student's intention to recommend OMT, yoga, medications, or other interventions for future patients. Evidence is needed to explore what role evidence-based medicine articles play in creating a treatment plan for patients with chronic LBP, for example, and how much second-hand experiences play a role in prescription frequency (ie, supervising physician or other colleague's experiences and recommendations during medical school or residency). This information would help elucidate the psychology behind prescription or recommendation of adjunctive treatment modalities to conventional medicine.

Consideration should be taken between intent to prescribe and ability to prescribe. The medical students in the current study did not have the ability to prescribe. This study should be reproduced with practicing physicians to further explore the effect of experience and evidence-based research knowledge on prescription. Such a study would better capture the reality of practice that students may not foresee, such as restrictions to practice OMT. These aspects could modify the psychology of prescribing behavior. For similar reasons, this study should be replicated at all medical education institutions that have clinicians who can prescribe.

A further challenge, especially for third- and fourthyear osteopathic medical students who are studying at clinical sites with real patients, is their preceptor role models who may dissuade them from using their OMT skills or may not use or recommend either OMT or yoga themselves. Thus, students may be influenced to not use OMT if their preceptors do not or will not allow them to use it.
The survey in the current study was not validated against a reference or criterion standard. However, the survey was constructed with face validity-it covers the content it purports to measure. The accuracy of the survey could be improved by adding some negative statements that parallel the positive statements to assess internal consistency of the responders.

Owing to the format of administration of this study, self-selection bias could have led to a skewed positive perspective on the recommendation of OMT or yoga. Some courses require surveys to be filled out in order for grades to be released to students or give incentives such as an extra point toward the final grade. No incentives to participate were used for this study, which may have unintentionally fostered self-selection bias. The fact that there were some participants who did not have positive experiences with yoga or OMT, yet chose to fill out the survey, does not support the concern that only those who had good experiences responded. Interestingly, although having a negative experience decreased the likelihood of future recommendation, not all participants who had negative experiences refrained from prescribing OMT or yoga to the patient in the vignette. So, having a positive or negative experience alone does not predict future prescribing practices.

Overall, these findings are vital to comprehensive medical education curricula. Although previous scales have been used to assess attitudes toward integrative medicine (eg, IMAQ and CHBQ), our results may be the first to assess factors that can be integrated early in the medical curriculum to affect future prescription. This study has implications for single accreditation system programs to provide opportunities for experience in, and review of, research articles on therapeutic modalities such as OMT and yoga, which would likely result in increased prescription and improved access to care for patients with chronic LBP. Medical educators should note that in some cases, these 2 approaches to gaining information (ie, personal experience and reading literature) about these modalities can and should be synergistic. The highest percentage of 
participants who recommended OMT or yoga were those who had both personal experience and read articles on the given modality. This finding aligns with the 2 key elements of modern evidence-based medicine: a focus on both clinical expertise and evidence born from research. ${ }^{25,26}$ Sackett et $\mathrm{al}^{25}$ integrated another aspect to the practice of medicine-the "patient's unique values and circumstance." This addition echoes modern medicine's move to empower patients. ${ }^{27}$ Empowered patients with chronic LBP who value evidence-supported practices such as OMT or yoga have a better chance at accessing those services if their physicians prescribe them.

Based on the results of this survey, it would seem that postgraduate institutions that require competency in OMT and expect application of osteopathic principles and practice in patient care should require familiarity with research articles that support its use. It is unknown, however, whether the results of this survey have external validity to postgraduate physicians and their trainers. Further research is needed to validate this survey and use it in other populations of health care providers whose scope of practice allows them to recommend OMT or yoga.

\section{Conclusion}

The results of this survey-based study indicate that personal experience and reading research articles that support the use of OMT or yoga improve the likelihood of osteopathic medical students recommending these modalities for their future patients with chronic LBP. All 4 null hypotheses were rejected by the data. Such results are important to understand, as they affect the structure of osteopathic medical education. If osteopathic institutions and educators expect future osteopathic physicians to provide patient access to OMT for chronic LBP and other conditions for which it is indicated, they must continue to offer both experiences and evidence-based research articles to osteopathic medical students.

\section{Author Contributions}

Drs Seffinger, Hurwitz, Quiamas, and Mervyn-Cohen provided substantial contributions to conception and design, acquisition of data, or analysis and interpretation of data; Drs Seffinger and Hurwitz and Student Doctor Kitch drafted the article or revised it critically for important intellectual content; all authors gave final approval of the version of the article to be published; and all authors agree to be accountable for all aspects of the work in ensuring that questions related to the accuracy or integrity of any part of the work are appropriately investigated and resolved.

\section{References}

1. Barnes PM, Bloom B, Nahin RL. Complementary and alternative medicine use among adults and children: United States, 2007. Natl Health Stat Report. 2008;12:1-23.

2. Clarke TC, Black LI, Stussman BJ, Barnes PM, Nahin RL. Trends in the use of complementary health approaches among adults: United States, 2002-2012. Natl Health Stat Report. 2015;(79):1-9.

3. National Center for Complementary and Alternative Medicine. Expanding Horizons of Healthcare: Five-Year Strategic Plan 2001-2005. Bethesda, MD: National Institutes of Health; 2000.

4. National Center for Complementary and Alternative Medicine. Exploring the Science of Complementary and Alternative Medicine: Third Strategic Plan 2011-2015. Bethesda, MD: National Institutes of Health; 2011.

5. Cowen VS, Cyr V. Complementary and alternative medicine in US medical schools. Adv Med Educ Pract. 2015;6:113-117. doi:10.2147/ AMEP.S69761

6. Joyce P, Wardle J, Zaslawski C. Medical student attitudes towards complementary and alternative medicine (CAM) in medical education: a critical review. J Complement Integr Med. 2016;13(4):333-345. doi:10.1515/jcim-2014-0053

7. Loh KP, Ghorab H, Clarke E, Conroy R, Barlow J. Medical students' knowledge, perceptions, and interest in complementary and alternative medicine. J Altern Complement Med. 2013;19(4):360-366. doi:10.1089/acm.2012.0014

8. Rees CE, Wearn AM, Dennis I, Amri H, Greenfield SM. Medical students' attitudes to complementary and alternative medicine: further validation of the IMAQ and findings from an international longitudinal study. Med Teach. 2009;31(2):125-132. doi:10.1080/01421590802139724

9. Schneider CD, Meek PM, Bell IR. Development and validation of IMAQ: Integrative Medicine Attitude Questionnaire. BMC Med Educ. 2003;3(5). doi:10.1186/1472-6920-3-5

10. Lie D, Boker J. Development and validation of the CAM health belief questionnaire $(\mathrm{CHBQ})$ and $\mathrm{CAM}$ use and attitudes amongst medical students. BMC Med Educ. 2004;4(2). doi:10.1186/1472-6920-4-2

11. Tilbrook HE, Cox $\mathrm{H}$, Hewitt $\mathrm{CE}$, et al. Yoga for chronic low back pain. a randomized trial. Ann Intern Med. 2011;155(9):569-578. doi:10.7326 /0003-4819-155-9-201111010-00003

12. Hotlzman S, Beggs RT. Yoga for chronic low back pain: a meta-analysis of randomized controlled trails. Pain Res Manag. 2013;18(5):257-272.

13. Cramer $H$, Lauche $R$, Haller $H$, Dobos $G$. A systematic review and meta-analysis of yoga for low back pain. Clin J Pain. 2013; 29 (5):450-460. doi:10.1097/AJP.0b013e31825e1492 
14. Sherman KJ, Cherkin DC, Wellman RD, et al. A randomized trial comparing yoga, stretching, and a self-care book for chronic low back pain. Arch Intern Med. 2011;171(22):2019-2026. doi:10.1001 /archinternmed.2011.524

15. Task Force on the Low Back Pain Clinical Practice Guidelines. American Osteopathic Association Guidelines for Osteopathic Manipulative Treatment (OMT) for Patients With Low Back Pain. J Am Osteopath Assoc. 2016;116(8):536-549, e31-e36. doi:10.7556/jaoa.2016.107

16. Hildago B. Evidence based orthopaedic manual therapy for patients with nonspecific low back pain: an integrative approach. Musculoskelet Rehabil. 2016;29(2):231-239. doi:10.3233/BMR-150619

17. Telles $\mathrm{S}$, Singh N. Science of the mind: ancient yoga texts and modern studies. Psychiatr Clin North Am. 2013;36(1):93-108. doi:10.1016/j. psc.2013.01.010

18. Streeter CC, Gerbarg PL, Saper RB, Ciraulo DA, Brown RP. Effects of yoga on the autonomic nervous system, gamma-aminobutyric-acid and allostasis in epilepsy, depression, and post-traumatic stress disorder. Med Hypotheses. 2012;78(5):571-579. doi:10.1016/j. mehy.2012.01.021

19. Licciardone JC, Kearns CM, Crow WT. Changes in biomechanical dysfunction and low back pain reduction with osteopathic manual treatment: results from the OSTEOPATHIC Trial. Man Ther. 2014;19 (4):324-330. doi:10.1016/j.math.2014.03.004

20. Licciardone JC, Kearns CM. Somatic dysfunction and its association with chronic low back pain, back-specific functioning, and general health: results from the OSTEOPATHIC Trial. J Am Osteopath Assoc. 2012;112(7):420-428.

21. Clark BC, Thomas JS, Walkowski SA, Howell JN. Biology of manual therapies. J Am Osteopath Assoc. 2012;112(9):617-629.

22. Zein-Hammoud M, Standley PR. Modeled osteopathic manipulative treatments: a review of their in vitro effects on fibroblast tissue preparations. J Am Osteopath Assoc. 2015;115(8):490-502. doi:10.7556/jaoa.2015.103

23. Clinical Guideline Subcommittee on Low Back Pain. American Osteopathic Association. American Osteopathic Association guidelines for osteopathic manipulative treatment (OMT) for patients with low back pain. J Am Osteopath Assoc. 2010;110(11):653-666.

24. Draper BB, Johnson JC, Fossum C, Chamberlain NR. Osteopathic medical students' beliefs about osteopathic manipulative treatment at 4 colleges of osteopathic medicine. J Am Osteopath Assoc. 2011;111 (11):615-630.

25. Sackett DL, Straus S, Richardson WS, et al. Evidence-Based Medicine. 3rd ed. London, England: Churchill Livingstone; 2000.

26. Friedland DJ, Go AS, Davoren JB, et al. Evidence-Based Medicine: A Framework for Clinical Practice. New York, NY: McGraw-Hill Education; 1998.

27. Saca-Hazboun $\mathrm{H}$. Empowering patients with knowledge: and update on trends in patient education. ONS Connect. 2007;22(5):8-13.

๑) 2018 American Osteopathic Association

\section{@The JAOA on Twitter}

Follow us at http://www.twitter.com/ The JAOA to get involved and stay connected with updates, highlights, and conversations about osteopathic medical research. 